\title{
Communication \\ Glucosylimidazolium Hydroxide: A Bench-Stable Carbohydrate Based Building Block
}

\author{
Johannes Schnegas ${ }^{1}$ and Stefan Jopp ${ }^{1,2, *(\mathbb{D})}$ \\ 1 Institute of Chemistry, University of Rostock, Albert-Einstein-Str. 3a, 18059 Rostock, Germany; \\ johannes.schnegas@uni-rostock.de \\ 2 Department Life, Light \& Matter, University of Rostock, Albert-Einstein-Str. 25, 18059 Rostock, Germany \\ * Correspondence: stefan.jopp@uni-rostock.de
}

check for updates

Citation: Schnegas, J.; Jopp, S. Glucosylimidazolium Hydroxide: A Bench-Stable Carbohydrate Based Building Block. Compounds 2021, 1, 154-163. https://doi.org/10.3390/ compounds1030014

Received: 11 October 2021

Accepted: 23 November 2021

Published: 7 December 2021

Publisher's Note: MDPI stays neutral with regard to jurisdictional claims in published maps and institutional affiliations.

Copyright: (C) 2021 by the authors. Licensee MDPI, Basel, Switzerland. This article is an open access article distributed under the terms and conditions of the Creative Commons Attribution (CC BY) license (https:// creativecommons.org/licenses/by/ $4.0 /)$.

\begin{abstract}
Hygroscopic effects in ionic liquids and salts in general, and how to suppress said hygroscopy, often needs to be considered during the everyday work routine. Chemicals that decompose, undergo hydrolysis or in any way change their composition when exposed to air are generally not considered to be bench-stable. In this study, we synthesized a low-hygroscopic, bench-stable carbohydrate-based hydroxide salt. This new product was synthesized in an optimized three-step procedure with $91 \%$ overall yield. Its worth as a building block was proven through the reaction with different natural acids, leading to new carbohydrate-based ionic liquids (CHILs) in the process.
\end{abstract}

Keywords: carbohydrate; ionic liquid; building block; nature-derived product; green chemistry

\section{Introduction}

Ionic liquids, organic ionic molecules with a melting point below $100{ }^{\circ} \mathrm{C}[1]$, have become a well-known class of compounds since their rise in popularity in the early 1990s. While ionic liquids have since evolved into several, often task-specific, sub-classes, such as room-temperature ionic liquids (RTILs) [2], polymerized ionic liquids (PILs) [3] or magnetic ionic liquids (MILs) [4], our working group is specifically focused on carbohydrate-based ionic liquids (CHILs) [5].

Ionic liquids are often considered an aspect of green chemistry. Their "greenness", however, is exclusively based on their remarkably low vapor pressure and flammability, which sets them apart from the common organic solvents. It has since become apparent that their favorable properties come with a price: many ionic liquids are produced from fossil fuels and have been found to be (eco)toxic [6-8]. However, our group found that ionic liquids based on glucosides and pentoses, thus originating from re-growing resources, exhibit a very low cytotoxicity [9]. Besides this striking potential as "greener" ionic liquids, several working groups have already proven many potential applications for CHILs, such as a positive influence on the rate and selectivity of Diels-Alder cycloadditions [10] or the ability to adsorb $\mathrm{Pb}^{2+}$ and $\mathrm{Cd}^{2+}$ ion from an aqueous solution [11,12].

As part of this ongoing topic, our working group began to examine the potential of CHILs as alternatives for the dissolution of cellulose. Cellulose is important in a broad variety of applications, including paper products, fibers and pharmaceuticals [13,14]. Among the currently known techniques for the dissolution of cellulose are aqueous sodium hydroxide with urea as additive [15], aqueous tetrabutylphosphonium hydroxide [16] and the ionic liquid [BMIM] acetate [17]. There is also a single example using a gluconic-acidbased ionic liquid [18].

Our group aimed to combine the aforementioned techniques and synthesized both tetrabutylphosphonium- and tetrabutylammonium glucuronate in full conversion using an aqueous solution of tetrabutylphosphonium- or tetrabutylammonium hydroxide and glucuronic acid, according to the literature [19]. We then produced a series of aqueous 
solutions ranging from 10 to 80 mass percent of the glucoronate salts. However, not even 0.5 mass percent of microcrystalline cellulose (particle size $20 \mu \mathrm{m}$ ) is soluble in these solutions. Using these salts as molten mass leads to decomposition, as the melting points of $125^{\circ} \mathrm{C}\left(\mathrm{PBu}_{4}\right.$ glucuronate $)$ and $145^{\circ} \mathrm{C}\left(\mathrm{NBu}_{4}\right.$ glucuronate $)$ are too high for cellulose.

These results led to the main part of this communication, in which we synthesized a glucose-based imidazolium hydroxide, since all of these structural elements are known in the aforementioned current techniques for the dissolution of cellulose. Other known carbohydrate-based ionic liquids with a cationic imidazolium moiety are based on galactose, ribose and isosorbide, and usually contain protecting groups (Figure 1) [20,21]. Since a common disadvantage of CHILs are their multistep syntheses, combined with often moderate yields, we focused on a short and optimized reaction procedure, thus dropping the usage of protecting groups.

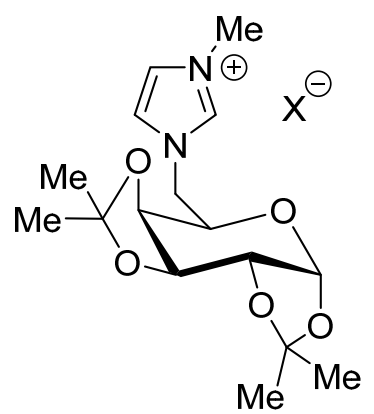

$\mathrm{X}=\mathrm{I}, \mathrm{OTf}, \mathrm{NTf}_{2}, \mathrm{PF}_{6}, \mathrm{BF}_{4}$ [20]<smiles>[X]C[n+]1ccn(C)c1</smiles>

$\mathrm{X}=\mathrm{Br}, \mathrm{I}, \mathrm{OTs}, \mathrm{NTf}_{2}, \mathrm{PF}_{6}, \mathrm{BF}_{4}$ [20]

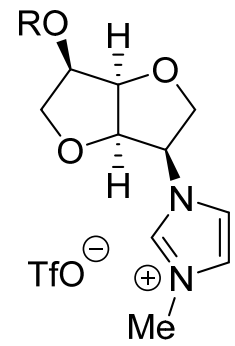

$\mathrm{R}=\mathrm{H}, \mathrm{Ac}, \mathrm{Bn}$ [21]

Figure 1. Examples of known CHILs containing a cationic imidazolium moiety.

\section{Materials and Methods}

\subsection{General Information}

All reagents and solvents were purchased from commercial sources and used as received without further purification, if not stated otherwise. The NMR spectra were recorded on a Bruker AVANCE $300 \mathrm{III}$ spectrometer (Bruker, Billerica, MA, USA). $\mathrm{CDCl}_{3}$ was calibrated as $7.27 \mathrm{ppm}\left({ }^{1} \mathrm{H}\right)$ and $77.00 \mathrm{ppm}\left({ }^{13} \mathrm{C}\right)$. DMSO-d 6 was calibrated as $2.49 \mathrm{ppm}$ $\left({ }^{1} \mathrm{H}\right)$ and $39.50 \mathrm{ppm}\left({ }^{13} \mathrm{C}\right) . \mathrm{CD}_{3} \mathrm{OD}$ was calibrated as $3.31 \mathrm{ppm}\left({ }^{1} \mathrm{H}\right)$ and $49.00 \mathrm{ppm}\left({ }^{13} \mathrm{C}\right)$. $\mathrm{D}_{2} \mathrm{O}$ was calibrated as $4.80 \mathrm{ppm}\left({ }^{1} \mathrm{H}\right)$. All $\delta$ values are in ppm and all $\mathrm{J}$ values are in $\mathrm{Hz}$. ESI-MS were measured with an Agilent 1200/6210 Time-of-Flight LC-MS (Agilent, Santa Clara, CA, USA). The measurements of thermogravimetric analysis (TGA) and differential scanning calorimetry (DSC) were performed on a Mettler Toledo DSC 823e instrument (Mettler Toledo, Columbus, $\mathrm{OH}$, USA) with a heating rate of $10^{\circ} \mathrm{C} / \mathrm{min}$ under an argon flow of $100 \mathrm{~mL} / \mathrm{min}$. The X-Ray crystal structure was measured with a Bruker-AXS Apex Kappa-II Single Crystal Diffractometer (Bruker, Billerica, MA, USA). All further crystal data can be accessed through the Cambridge Crystallographic Data Centre with number 2121301. The starting material 1 (CAS: 97-30-3) was commercially supplied by TCI.

\subsection{Experimental}

\subsubsection{Methyl 6-Iodo- $\alpha$-D-Glucopyranoside 2}

Methyl $\alpha$-D-glucopyranoside 1 (1.01 g; $5.2 \mathrm{mmol})$, triphenylphosphine (2.05 g; $7.8 \mathrm{mmol}$; 1.5 eq.) and imidazole ( $0.71 \mathrm{~g} ; 10.4 \mathrm{mmol} ; 2.0$ eq.) and iodine (1.98 g; $7.8 \mathrm{mmol} ; 1.5$ eq.) were refluxed in THF $(30 \mathrm{~mL})$ for $4 \mathrm{~h}$. The resulting solid was filtered off, the solvent was removed via rotary evaporator and the product was obtained as a white solid (1.45 g; 92\%) after silica-gel column chromatography (chloroform/methanol 12:1). This procedure was altered from the literature [22]. The work-up procedure was changed from reverse-phase octadecyl capped silica-gel column chromatography with water/methanol followed by a further recrystallization in ethanol to silica-gel column chromatography with chloroform/methanol. 
m.p.: $148-149^{\circ} \mathrm{C}$ (lit.: $\left.147-148^{\circ} \mathrm{C}\right) .[\alpha]_{D}^{25}=+94.2\left(\mathrm{c}=1.0, \mathrm{H}_{2} \mathrm{O}\right)($ lit.: $+99.7, \mathrm{c}=2.0$, $\left.\mathrm{CHCl}_{3}\right) .{ }^{1} \mathrm{H}$ NMR $\left(300 \mathrm{MHz}, \mathrm{DMSO}-\mathrm{d}_{6}\right): \delta=2.87-2.95(\mathrm{~m}, 1 \mathrm{H}) ; 3.16-3.27(\mathrm{~m}, 3 \mathrm{H}) ; 3.31(\mathrm{~s}$, $\left.3 \mathrm{H}, \mathrm{OCH}_{3}\right) ; 3.34-3.42(\mathrm{~m}, 1 \mathrm{H}) ; 3.50-3.57(\mathrm{~m}, 1 \mathrm{H}) ; 4.54\left(\mathrm{~d}, 1 \mathrm{H},{ }^{3} \mathrm{~J}=3.65, \mathrm{H}-1\right) ; 4.78(\mathrm{~d}, 1 \mathrm{H}$, $\left.{ }^{3} \mathrm{~J}=6.43, \mathrm{OH}\right) ; 4.86\left(\mathrm{~d}, 1 \mathrm{H},{ }^{3} \mathrm{~J}=4.99, \mathrm{OH}\right) ; 5.17\left(\mathrm{~d}, 1 \mathrm{H},{ }^{3} \mathrm{~J}=5.83, \mathrm{OH}\right) .{ }^{13} \mathrm{C}$ NMR $(75 \mathrm{MHz}$, DMSO-d 6$): \delta=9.5(\mathrm{C}-6) ; 54.6\left(\mathrm{OCH}_{3}\right) ; 70.9,71.9,72.7,74.1$ (C-2, C-3, C-4, C-5); $99.8(\mathrm{C}-1)$. (Figure S1).

\subsubsection{1-(Methyl 6-Deoxy- $\alpha$-D-Glucopyranosid-6-yl)-3-Methylimidazolium Iodide 3}

Compound 2 ( $3.95 \mathrm{~g} ; 13.0 \mathrm{mmol})$ was dissolved in N-methylimidazol $(26 \mathrm{~mL}, 2 \mathrm{~mL} / \mathrm{mmol})$ and stirred at $125^{\circ} \mathrm{C}$ for $6 \mathrm{~h}$. Water $(50 \mathrm{~mL})$ was added and the aqueous solution was washed thoroughly with dichloromethane $(20 \times 20 \mathrm{~mL})$. The water was then removed via rotary evaporator and the resulting oil was washed with ethyl acetate $(10 \times 15 \mathrm{~mL})$ until crystallization. The product was obtained as a yellow solid (5.01 $\mathrm{g} ;>99 \%)$.

m.p.: $172-173{ }^{\circ} \mathrm{C} .[\alpha]_{D}^{25}=+68.3\left(\mathrm{c}=1.0, \mathrm{H}_{2} \mathrm{O}\right) .{ }^{1} \mathrm{H}$ NMR $\left(300 \mathrm{MHz}, \mathrm{D}_{2} \mathrm{O}\right): \delta=3.26-3.32$ $(\mathrm{m}, 1 \mathrm{H}) ; 3.31\left(\mathrm{~s}, 3 \mathrm{H}, \mathrm{OCH}_{3}\right) ; 3.63\left(\mathrm{dd}, 1 \mathrm{H},{ }^{3} \mathrm{~J}=9.77,{ }^{3} \mathrm{~J}=3.76, \mathrm{H}-2\right) ; 3.72-3.78(\mathrm{~m}, 1 \mathrm{H})$; $3.94-3.98(\mathrm{~m}, 1 \mathrm{H}) ; 4.00\left(\mathrm{~s}, 3 \mathrm{H}, \mathrm{NCH}_{3}\right) ; 4.50\left(\mathrm{dd}, 1 \mathrm{H},{ }^{2} \mathrm{~J}=14.60,{ }^{3} \mathrm{~J}=7.30, \mathrm{H}-6 \mathrm{a}\right) ; 4.69$ (dd, $1 \mathrm{H}$, $\left.{ }^{2} \mathrm{~J}=14.60,{ }^{3} \mathrm{~J}=2.54, \mathrm{H}-6 \mathrm{~b}\right) ; 4.89\left(\mathrm{~d}, 1 \mathrm{H},{ }^{3} \mathrm{~J}=3.74, \mathrm{H}-1\right) ; 7.55\left(\mathrm{~d}, 1 \mathrm{H},{ }^{3} \mathrm{~J}=1.95, \mathrm{H}_{\mathrm{Ar}}\right) ; 7.64(\mathrm{~d}$, $\left.1 \mathrm{H},{ }^{3} \mathrm{~J}=1.95, \mathrm{H}_{\mathrm{Ar}}\right) ; 8.89\left(\mathrm{~s}, 1 \mathrm{H}, \mathrm{H}_{\mathrm{Ar}}\right) .{ }^{13} \mathrm{C} \mathrm{NMR}\left(75 \mathrm{MHz}, \mathrm{D}_{2} \mathrm{O}\right): \delta=36.1\left(\mathrm{NCH}_{3}\right) ; 49.9(\mathrm{C}-6)$; $55.2\left(\mathrm{OCH}_{3}\right) ; 69.4,70.5,71.0,72.9$ (C-2, C-3, C-4, C-5); $99.3(\mathrm{C}-1) ; 123.2,123.6,137.0\left(\mathrm{CH}_{\mathrm{Ar}}\right)$. HRMS (ESI, $m / z$ ): Calculated for $\mathrm{C}_{11} \mathrm{H}_{19} \mathrm{~N}_{2} \mathrm{O}_{5}^{+}, 259.1294 ;$ measured 259.1299. Calculated for $\mathrm{I}^{-}, 126.9050$; measured 126.9047. (Figure S2).

\subsubsection{1-(Methyl 6-Deoxy- $\alpha$-D-Glucopyranosid-6-yl)-3-Methylimidazolium Hydroxide 4}

Amberlite ${ }^{\circledR}$ IRN-78 ion exchange gel was set up like a column chromatography and 3 ( $2.05 \mathrm{~g} ; 5.3 \mathrm{mmol}$ ) was eluted with water. All test tubes were tested with pH 0-14 test strips and all alkaline test tubes were collected. The product was obtained as a yellow solid $(1.46 \mathrm{~g} ;>99 \%)$ after the removal of water via rotary evaporator.

m.p.: no melting until decomposition at $139-142{ }^{\circ} \mathrm{C} .[\alpha]_{D}^{25}=+89.4\left(\mathrm{c}=1.0, \mathrm{H}_{2} \mathrm{O}\right) \cdot{ }^{1} \mathrm{H}$ $\operatorname{NMR}\left(300 \mathrm{MHz}, \mathrm{D}_{2} \mathrm{O}\right): \delta=3.18\left(\mathrm{dd}, 1 \mathrm{H},{ }^{3} \mathrm{~J}=9.94{ }^{3} \mathrm{~J}=8.79, \mathrm{H}-4\right) ; 3.23\left(\mathrm{~s}, 3 \mathrm{H}, \mathrm{OCH}_{3}\right) ; 3.52$ $\left(\mathrm{dd}, 1 \mathrm{H},{ }^{3} \mathrm{~J}=9.73,{ }^{3} \mathrm{~J}=3.77, \mathrm{H}-2\right) ; 3.64\left(\mathrm{dd}, 1 \mathrm{H},{ }^{3} \mathrm{~J}=9.70,{ }^{3} \mathrm{~J}=8.79, \mathrm{H}-3\right) ; 3.85$ (ddd, 1H, $\left.{ }^{3} \mathrm{~J}=9.94,{ }^{3} \mathrm{~J}=7.45,{ }^{3} \mathrm{~J}=2.50, \mathrm{H}-5\right) ; 3.92\left(\mathrm{~s}, 3 \mathrm{H}, \mathrm{NCH}_{3}\right) ; 4.40\left(\mathrm{dd}, 1 \mathrm{H},{ }^{2} \mathrm{~J}=14.59,{ }^{3} \mathrm{~J}=7.48\right.$, H-6a); 4.62 (dd, 1H, $\left.{ }^{2} \mathrm{~J}=14.58,{ }^{3} \mathrm{~J}=2.52, \mathrm{H}-6 \mathrm{~b}\right) ; 4.78-4.80$ (m, 1H, H-1); 7.47 (d, 1H, ${ }^{3} \mathrm{~J}=1.99$, $\left.\mathrm{H}_{\mathrm{Ar}}\right) ; 7.57\left(\mathrm{~d}, 1 \mathrm{H},{ }^{3} \mathrm{~J}=2.00, \mathrm{H}_{\mathrm{Ar}}\right) .{ }^{13} \mathrm{C} \mathrm{NMR}\left(75 \mathrm{MHz}, \mathrm{D}_{2} \mathrm{O}\right): \delta=35.7\left(\mathrm{NCH}_{3}\right) ; 50.0(\mathrm{C}-6)$; $54.9\left(\mathrm{OCH}_{3}\right) ; 70.0,70.9,71.2,73.5$ (C-2, C-3, C-4, C-5); $99.5(\mathrm{C}-1) ; 123.1,123.4\left(\mathrm{CH}_{\mathrm{Ar}}\right)$. HRMS (ESI, $m / z$ ): Calculated for $\mathrm{C}_{11} \mathrm{H}_{19} \mathrm{~N}_{2} \mathrm{O}_{5}^{+}, 259.1294$; measured 259.1296. (Figure S3).

\subsubsection{1-(Methyl 6-Deoxy- $\alpha$-D-Glucopyranosid-6-yl)-3-Methylimidazolium Glucuronate 6a}

Compound 4 (340 mg; $1.1 \mathrm{mmol})$ was dissolved in water $(15 \mathrm{~mL})$. D-Glucuronic acid (256 mg; $1.32 \mathrm{mmol}, 1.2$ eq.) was added slowly. The solution was stirred vigorously for $24 \mathrm{~h}$. The water was removed via rotary evaporator and the resulting oil was dissolved in methanol. The remaining insoluble D-Glucuronic acid was filtered off and the solvent was removed via rotary evaporator again to obtain the product as an off-white solid (584 $\mathrm{mg}$; $98 \%)$.

m.p.: $106-111^{\circ} \mathrm{C} .[\alpha]_{D}^{25}=+60.4\left(\mathrm{c}=1.0, \mathrm{H}_{2} \mathrm{O}\right)$. Due to the $\alpha / \beta$-anomeric mixture of the glucuronate anion, we will list only major proton NMR signals. ${ }^{1} \mathrm{H} \mathrm{NMR}(300 \mathrm{MHz}$, $\left.\mathrm{D}_{2} \mathrm{O}\right): \delta=3.37\left(\mathrm{~s}, 3 \mathrm{H}, \mathrm{OCH}_{3}\right) ; 3.93\left(\mathrm{~s}, 3 \mathrm{H}, \mathrm{NCH}_{3}\right) ; 4.83\left(\mathrm{~d}, 1 \mathrm{H},{ }^{3} \mathrm{~J}=3.83, \alpha\right.$-H-1-cation); 5.26 $\left(\mathrm{d}, 0.76 \mathrm{H},{ }^{3} \mathrm{~J}=3.71, \alpha-\mathrm{H}\right.$-1-anion); $7.49\left(\mathrm{~d}, 1 \mathrm{H},{ }^{3} \mathrm{~J}=1.81, \mathrm{H}_{\mathrm{Ar}}\right) ; 7.58\left(\mathrm{~d}, 1 \mathrm{H},{ }^{3} \mathrm{~J}=1.82, \mathrm{H}_{\mathrm{Ar}}\right) ; 8.82$ (s, $\left.1 \mathrm{H}, \mathrm{H}_{\mathrm{Ar}}\right) .{ }^{13} \mathrm{C} \mathrm{NMR}\left(75 \mathrm{MHz}, \mathrm{D}_{2} \mathrm{O}\right): \delta=35.8\left(\mathrm{NCH}_{3}\right) ; 48.9$ (C-6-cation); $55.0\left(\mathrm{OCH}_{3}\right) ; 69.4$, $70.4,71.0,71.3,71.6,71.7,72.0,72.5,72.9,74.0,75.5,76.0$ (all C-2 to C-5 signals); $92.1,95.9$ (C-1-anion); 99.3 (C-1-cation); 123.2, 123.5, $137.0\left(\mathrm{CH}_{\mathrm{Ar}}\right)$; 175.4. 176.3 (C=O). HRMS (ESI, $m / z$ ): Calculated for $\mathrm{C}_{11} \mathrm{H}_{19} \mathrm{~N}_{2} \mathrm{O}_{5}^{+}$, 259.1294; measured 259.1294. Calculated for $\mathrm{C}_{6} \mathrm{H}_{9} \mathrm{O}_{7}^{-}$, 193.0354; measured 193.0357. (Figure S4). 
2.2.5. 1-(Methyl 6-Deoxy- $\alpha$-D-Glucopyranosid-6-yl)-3-Methylimidazolium L-Alaninate 6b

Compound 4 (340 mg; $1.1 \mathrm{mmol})$ was dissolved in water $(15 \mathrm{~mL})$. L-Alanine (118 mg; $1.32 \mathrm{mmol}, 1.2$ eq.) was added slowly. The solution was stirred vigorously for $24 \mathrm{~h}$. The water was removed via rotary evaporator and the resulting oil was dissolved in methanol. The remaining insoluble L-alanine was filtered off and the solvent was removed via rotary evaporator again to obtain the product as a yellow oil (378 $\mathrm{mg} ; 99 \%)$.

$[\alpha]_{D}^{25}=+75.9\left(\mathrm{c}=1.5, \mathrm{H}_{2} \mathrm{O}\right) .{ }^{1} \mathrm{H}$ NMR $\left(300 \mathrm{MHz}, \mathrm{D}_{2} \mathrm{O}\right): \delta=1.26\left(\mathrm{t}, 3 \mathrm{H},{ }^{3} \mathrm{~J}=7.07, \mathrm{CH}_{3}\right) ;$ $3.21\left(\mathrm{dd}, 1 \mathrm{H},{ }^{3} \mathrm{~J}=10.02,{ }^{3} \mathrm{~J}=8.95\right) ; 3.25\left(\mathrm{~s}, 3 \mathrm{H}, \mathrm{OCH}_{3}\right) ; 3.37\left(\mathrm{q}, 1 \mathrm{H},{ }^{3} \mathrm{~J}=7.18, \mathrm{CH}\right) ; 3.55(\mathrm{dd}$, $\left.1 \mathrm{H},{ }^{3} \mathrm{~J}=9.78,{ }^{3} \mathrm{~J}=3.80, \mathrm{H}-2\right) ; 3.66-3.72(\mathrm{~m}, 1 \mathrm{H}) ; 3.87-3.92(\mathrm{~m}, 1 \mathrm{H}) ; 3.93\left(\mathrm{~s}, 3 \mathrm{H}, \mathrm{NCH}_{3}\right)$; $4.43\left(\mathrm{dd}, 1 \mathrm{H},{ }^{2} \mathrm{~J}=14.62,{ }^{3} \mathrm{~J}=7.36, \mathrm{H}-6 \mathrm{a}\right) ; 4.63\left(\mathrm{dd}, 1 \mathrm{H},{ }^{2} \mathrm{~J}=14.60,{ }^{3} \mathrm{~J}=2.57, \mathrm{H}-6 \mathrm{~b}\right) ; 4.82$ $\left(\mathrm{d}, 1 \mathrm{H},{ }^{3} \mathrm{~J}=3.76, \mathrm{H}-1\right) ; 7.49\left(\mathrm{~d}, 1 \mathrm{H},{ }^{3} \mathrm{~J}=2.01, \mathrm{H}_{\mathrm{Ar}}\right) ; 7.58\left(\mathrm{~d}, 1 \mathrm{H},{ }^{3} \mathrm{~J}=2.02, \mathrm{H}_{\mathrm{Ar}}\right) .{ }^{13} \mathrm{C}$ NMR $\left(75 \mathrm{MHz}, \mathrm{D}_{2} \mathrm{O}\right): \delta=17.1\left(\mathrm{CH}_{3}\right) ; 33.0\left(\mathrm{NCH}_{3}\right) ; 46.1(\mathrm{CH}) ; 47.1(\mathrm{C}-6), 52.2\left(\mathrm{OCH}_{3}\right) ; 66.7,67.7$, 68.3, 70.1 (C-2, C-3, C-4, C-5); 96.6 (C-1); 120.4, $120.7\left(\mathrm{CH}_{\mathrm{Ar}}\right) ; 180.8$ (C=O). HRMS (ESI, $m / z$ ): Calculated for $\mathrm{C}_{11} \mathrm{H}_{19} \mathrm{~N}_{2} \mathrm{O}_{5}^{+}$, 259.1294; measured 259.1291. Calculated for $\mathrm{C}_{3} \mathrm{H}_{6} \mathrm{NO}_{2}^{-}$, 88.0404; measured 88.0407. (Figure S5).

\subsubsection{1-(Methyl 6-Deoxy- $\alpha$-D-Glucopyranosid-6-yl)-3-Methylimidazolium Trans-Cinnamate 6c}

Compound 4 (340 mg; $1.1 \mathrm{mmol}$ ) was dissolved in water $(15 \mathrm{~mL})$. trans-Cinnamic acid (196 mg; $1.32 \mathrm{mmol}, 1.2$ eq.) was added slowly. The suspension was stirred vigorously for $24 \mathrm{~h}$. The water was removed via rotary evaporator and the resulting solid was washed with chloroform $(2 \times 10 \mathrm{~mL})$. The product was obtained as an off-white solid (407 mg; 91\%).

m.p.: $38-40{ }^{\circ} \mathrm{C} .[\alpha]_{D}^{25}=+62.9\left(\mathrm{c}=1.3, \mathrm{H}_{2} \mathrm{O}\right) .{ }^{1} \mathrm{HNMR}\left(300 \mathrm{MHz}, \mathrm{DMSO}-\mathrm{d}_{6}\right): \delta=2.87-2.83$ $(\mathrm{m}, 1 \mathrm{H}) ; 3.09\left(\mathrm{~s}, 3 \mathrm{H}, \mathrm{OCH}_{3}\right) ; 3.18\left(\mathrm{dd}, 1 \mathrm{H},{ }^{3} \mathrm{~J}=9.60,{ }^{3} \mathrm{~J}=3.70, \mathrm{H}-2\right) ; 3.43-3.49(\mathrm{~m}, 1 \mathrm{H}) ; 3.65(\mathrm{ddd}$, $\left.1 \mathrm{H},{ }^{3} \mathrm{~J}=9.66,{ }^{3} \mathrm{~J}=7.26,{ }^{3} \mathrm{~J}=2.71, \mathrm{H}-5\right) ; 3.86\left(\mathrm{~s}, 3 \mathrm{H}, \mathrm{NCH}_{3}\right) ; 4.33\left(\mathrm{dd}, 1 \mathrm{H},{ }^{2} \mathrm{~J}=14.23,{ }^{3} \mathrm{~J}=7.26, \mathrm{H}-6 \mathrm{a}\right)$; $4.51\left(\mathrm{dd}, 1 \mathrm{H},{ }^{2} \mathrm{~J}=14.11,{ }^{3} \mathrm{~J}=3.01, \mathrm{H}-6 \mathrm{~b}\right) ; 4.53\left(\mathrm{~d}, 1 \mathrm{H},{ }^{3} \mathrm{~J}=3.47, \mathrm{H}-1\right) ; 6.42\left(\mathrm{~d}, 1 \mathrm{H},{ }^{3} \mathrm{~J}=15.92, \mathrm{CH}\right)$; $7.21\left(\mathrm{~d}, 1 \mathrm{H},{ }^{3} \mathrm{~J}=15.94, \mathrm{CH}\right) ; 7.28-7.37\left(\mathrm{~m}, 3 \mathrm{H}, \mathrm{H}_{\mathrm{Ar}}\right) ; 7.50-7.53\left(\mathrm{~m}, 2 \mathrm{H}, \mathrm{H}_{\mathrm{Ar}}\right) ; 7.69-7.74(\mathrm{~m}, 2 \mathrm{H}$, $\left.\mathrm{H}_{\mathrm{Ar}}\right) ; 9.20\left(\mathrm{~s}, 1 \mathrm{H}, \mathrm{H}_{\mathrm{Ar}}\right) .{ }^{13} \mathrm{C} \mathrm{NMR}\left(75 \mathrm{MHz}, \mathrm{DMSO}-\mathrm{d}_{6}\right): \delta=35.7\left(\mathrm{NCH}_{3}\right) ; 50.1(\mathrm{C}-6) ; 54.5\left(\mathrm{OCH}_{3}\right)$; 69.7, 71.1, 71.7, 72.7 (C-2, C-3, C-4, C-5); 100.2 (C-1); 123.2, 127.2, 127.5, 128.4, 128.7, 136.1, 137.4, $137.5\left(\mathrm{CH}_{\mathrm{Ar}}\right) ; 169.9(\mathrm{C}=\mathrm{O})$. HRMS (ESI, $\left.m / z\right)$ : Calculated for $\mathrm{C}_{11} \mathrm{H}_{19} \mathrm{~N}_{2} \mathrm{O}_{5}^{+}$, 259.1294; measured 259.1292. Calculated for $\mathrm{C}_{9} \mathrm{H}_{7} \mathrm{O}_{2}{ }^{-}, 147.0452$; measured 147.0457. (Figure S6).

\subsubsection{1-(Methyl 6-Deoxy- $\alpha$-D-Glucopyranosid-6-yl)-3-Methylimidazolium Caprylate 6d}

Compound 4 (340 mg; $1.1 \mathrm{mmol})$ was dissolved in water $(15 \mathrm{~mL})$. Caprylic acid (209 $\mu \mathrm{L} ; 1.32 \mathrm{mmol}, 1.2 \mathrm{eq}$.) was added dropwise. The emulsion was stirred vigorously for $24 \mathrm{~h}$. The water was removed via rotary evaporator and the resulting oil was washed with $n$-heptane $(2 \times 10 \mathrm{~mL})$. The product was obtained as a yellow oil $(437 \mathrm{mg} ; 99 \%)$.

$[\alpha]_{D}^{25}=+27.3\left(\mathrm{c}=2.2, \mathrm{H}_{2} \mathrm{O}\right) .{ }^{1} \mathrm{H}$ NMR $\left(300 \mathrm{MHz}, \mathrm{DMSO}_{-} \mathrm{d}_{6}\right): \delta=0.84\left(\mathrm{t}, 3 \mathrm{H},{ }^{3} \mathrm{~J}=6.75\right.$, $\left.\mathrm{CH}_{3}\right) ; 1.21-1.26\left(\mathrm{~m}, 8 \mathrm{H}, 4 \times \mathrm{CH}_{2}\right) ; 1.36-1.45\left(\mathrm{~m}, 2 \mathrm{H}, \mathrm{CH}_{2}\right) ; 1.94\left(\mathrm{t}, 2 \mathrm{H},{ }^{3} \mathrm{~J}=7.37, \mathrm{CH}_{2}\right)$; $2.83-2.89(\mathrm{~m}, 1 \mathrm{H}) ; 3.08\left(\mathrm{~s}, 3 \mathrm{H}, \mathrm{OCH}_{3}\right) ; 3.15\left(\mathrm{dd}, 1 \mathrm{H},{ }^{3} \mathrm{~J}=9.58,{ }^{3} \mathrm{~J}=3.68, \mathrm{H}-2\right) ; 3.39-3.45$ $(\mathrm{m}, 1 \mathrm{H}) ; 3.62\left(\mathrm{ddd}, 1 \mathrm{H},{ }^{3} \mathrm{~J}=9.58,{ }^{3} \mathrm{~J}=7.42,{ }^{3} \mathrm{~J}=2.41, \mathrm{H}-5\right) ; 3.86\left(\mathrm{~s}, 3 \mathrm{H}, \mathrm{NCH}_{3}\right) ; 4.31(\mathrm{dd}, 1 \mathrm{H}$, ${ }^{2} \mathrm{~J}=14.20,{ }^{3} \mathrm{~J}=7.29$, H-6a); $4.49\left(\mathrm{dd}, 1 \mathrm{H},{ }^{2} \mathrm{~J}=14.54,{ }^{3} \mathrm{~J}=2.98, \mathrm{H}-6 \mathrm{~b}\right) ; 4.51\left(\mathrm{~d}, 1 \mathrm{H},{ }^{3} \mathrm{~J}=3.49\right.$, H1); $7.69-7.73\left(\mathrm{~m}, 2 \mathrm{H}, \mathrm{H}_{\mathrm{Ar}}\right) ; 8.89\left(\mathrm{~s}, 1 \mathrm{H}, \mathrm{H}_{\mathrm{Ar}}\right) .{ }^{13} \mathrm{C} \mathrm{NMR}\left(75 \mathrm{MHz}, \mathrm{DMSO}-\mathrm{d}_{6}\right): \delta=13.4\left(\mathrm{CH}_{3}\right)$; 26.1, 25.3, 28.2, 28.6, $30.8\left(\mathrm{CH}_{2}\right) ; 35.2\left(\mathrm{NCH}_{3}\right) ; 36.4\left(\mathrm{CH}_{2}\right) ; 49.6(\mathrm{C}-6) ; 54.0\left(\mathrm{OCH}_{3}\right) ; 69.2$, 70.7, 71.2, 72.1 (C-2, C-3, C-4, C-5); 99.7 (C-1); 122.7, $137.0\left(\mathrm{CH}_{\mathrm{Ar}}\right) ; 175.9$ (C=O). HRMS (ESI, $m / z)$ : Calculated for $\mathrm{C}_{11} \mathrm{H}_{19} \mathrm{~N}_{2} \mathrm{O}_{5}^{+}, 259.1294$; measured 259.1295. Calculated for $\mathrm{C}_{8} \mathrm{H}_{15} \mathrm{O}_{2}^{-}$, 143.1078; measured 143.1080. (Figure S7).

\section{Results and Discussion}

Using methyl $\alpha$-D-glucopyranoside 1 as the starting material, we first converted the primary $\mathrm{OH}$-group into an iodine leaving group using an Appel reaction as published by Madsen et al. [22]. The authors state that reverse-phase column chromatography followed by an additional recrystallization in ethanol is the best purification technique, yielding 
$77 \%$ of the product. However, after optimizing the reaction procedure and the work-up by simply using a silica gel column chromatography with chloroform and methanol as eluent mixture, we optimized the reaction to $92 \%$ yield, while still achieving a highly pure product (Scheme 1). This reaction has also been scaled up to a $7 \mathrm{~g}$ product scale with only a slight drop in the yield, to $85 \%$.

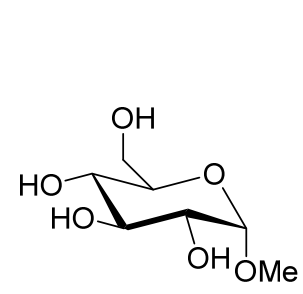

1

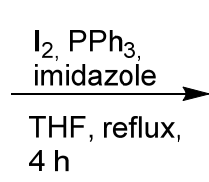

$4 \mathrm{~h}$

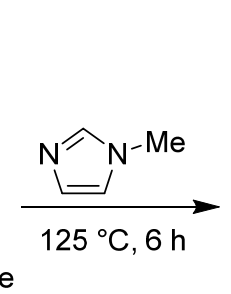

)

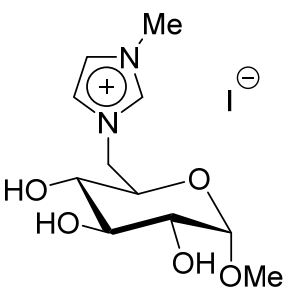

$3(>99 \%)$

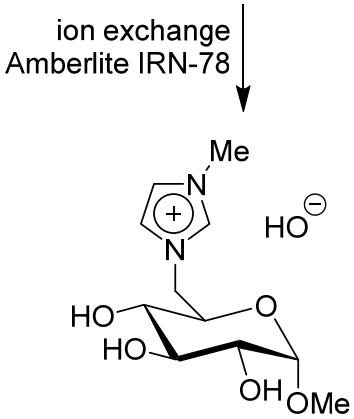

$4(>99 \%)$

Scheme 1. 3-step synthesis of glucosylimidazolium hydroxide 4 .

The iodinated compound 2 was then quarternized using $N$-methylimidazole in excess at $125{ }^{\circ} \mathrm{C}$ in full conversion. The iodide salt 3 was purified through extraction and washing.

A slow crystallization from a heated solution of 3 in acetone also leads to the formation of colorless needles, which we analyzed with X-ray analysis (Figure 2). The crystal structure confirms a chair conformation of the glucopyranoside, the alpha anomeric methoxy group, the bonding of the planar imidazolium heterocycle to the C-6 of the carbohydrate and, lastly, the iodide anion.

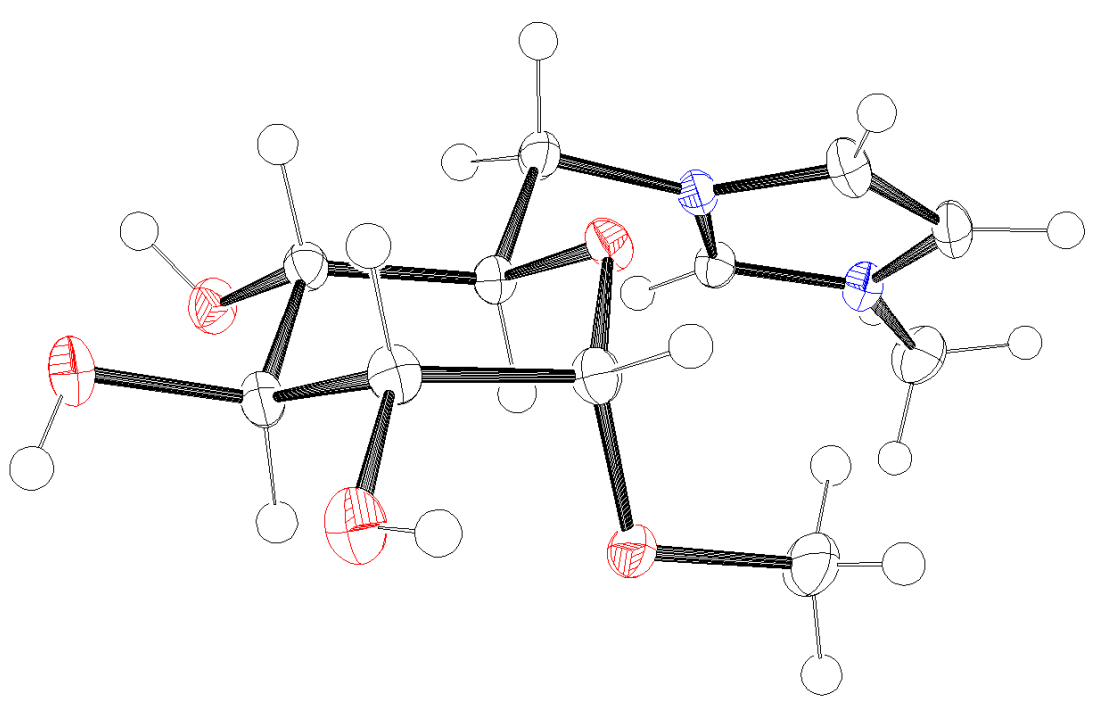

Figure 2. ORTEP of 3 [23]. 
As the last step, the iodide anion was exchanged to a hydroxide anion (Scheme 1). At first, we converted 3 into 4 using an aqueous silver(I) oxide solution, which leads to the precipitation of silver(I) iodide and leaves the glucosylimidazolium cation and a hydroxide anion as the remaining ions in the solution. While this procedure, when used for other ionic liquids by Yoshizawa-Fujita et al., proceeded smoothly and resulted in $85 \%$ yield after purification [24], the product was still visibly impure (dark brown color), most likely due to remaining silver. The NMR, however, showed no other organic impurities. Furthermore, silver(I) oxide is a costly reagent. Thus, we later used the ion exchange resin Amberlite ${ }^{\circledR}$ IRN-78 with high efficiency. The product is obtained in yields ranging from 87 to $99 \%$ and the exchange resin can be recycled using a sodium hydroxide solution (Scheme 1). To prove the successful synthesis of 4 , the iodide anion needs to be completely exchanged with the hydroxide anion. Since the NMRs of $\mathbf{3}$ and $\mathbf{4}$ are basically identical (see supporting information), besides minor shifts, the easiest method of proving the absence of iodide is by adding a few drops a silver(I) nitrate solution: No precipitate means no remaining iodide. If iodide remains, which was sometimes the case in minuscule amounts, the sample can simply be run over the recycled exchange resin again.

As mentioned in the introductory part of this short communication, the initial idea behind the synthesis of 4 was its use for the dissolution of cellulose. Compound 4 combines several structural elements, which previously led to the successful dissolution of cellulose, namely, a carbohydrate core [18], an imidazolium moiety [17] and a hydroxide anion $[15,16]$. Unfortunately, similar to the previously mentioned $\mathrm{PBu}_{4}$ glucuronate and $\mathrm{NBu}_{4}$ glucuronate, 4 proved to be incapable of fully dissolving even 0.5 mass percent of microcrystalline cellulose (particle size $20 \mu \mathrm{m}$ ). However, slight differences were found in the microscopic analysis of the suspensions, which showed fewer remaining cellulose crystals in the $40 \%$ aqueous solution of 4 (Figure 3).
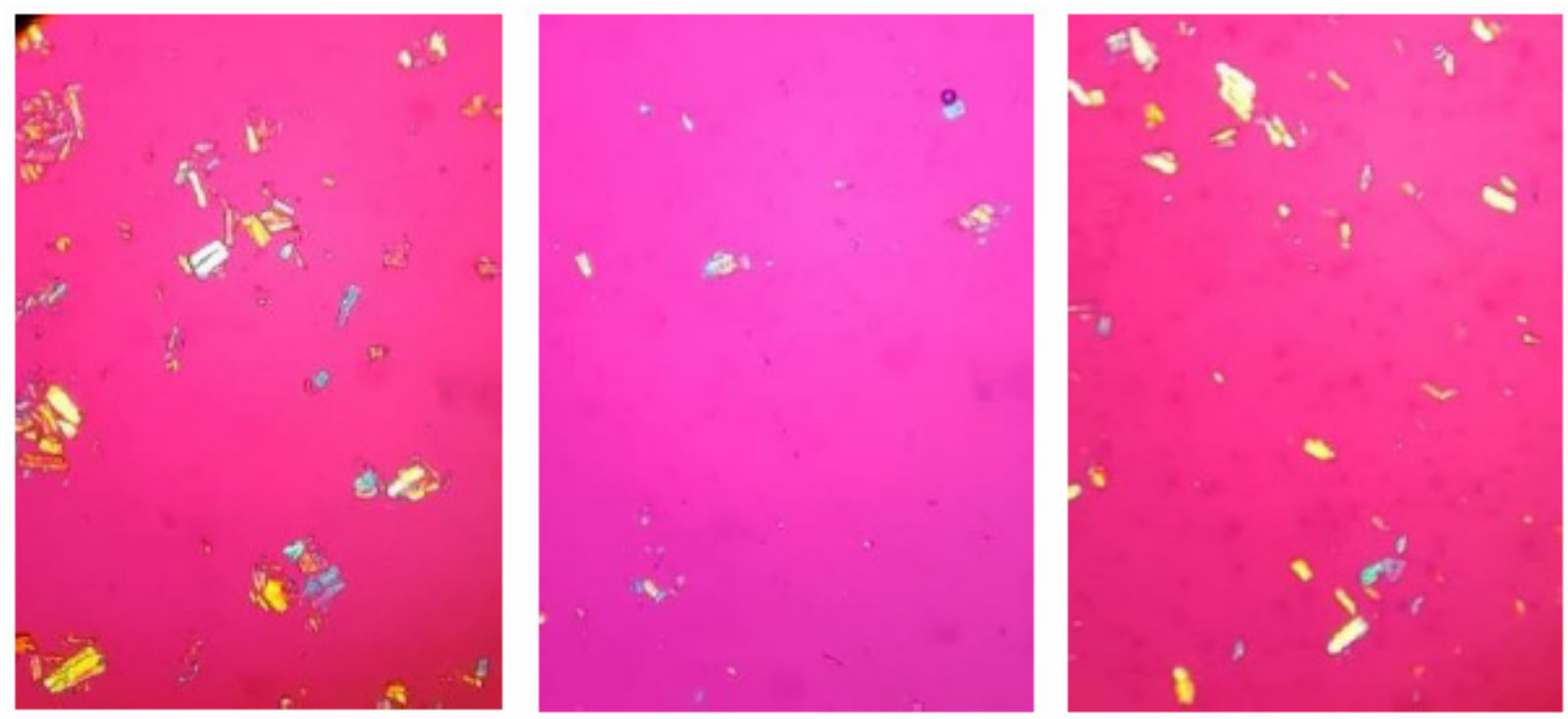

Figure 3. Microscopic analysis of 0.5 mass percent of cellulose in aqueous solution of 4 (from left to right: $10 \%, 40 \%, 60 \%$ ).

Due to the nature of the project, we always kept 4 as a $50 \%$ aqueous solution for our cellulose dissolution experiments. We furthermore suspected that the product will be unstable, as several other neat imidazolium-based hydroxides are [25], and hygroscopic, since this is generally common for hydroxide salts such as sodium or potassium hydroxide and was common for other similar CHILs of our recent project [26].

After drying 4 for the first time for its thermal analysis, to our surprise, we observed no initial hygroscopy. To further test this, we kept 4 in an open vial for 1 week, observing neither any hygroscopy nor any changes in the NMR (Figure 4). However, a clear deliquescence can be observed afterwards. However, this does not affect the overall stability of the product and the salt may simply be dried again. 


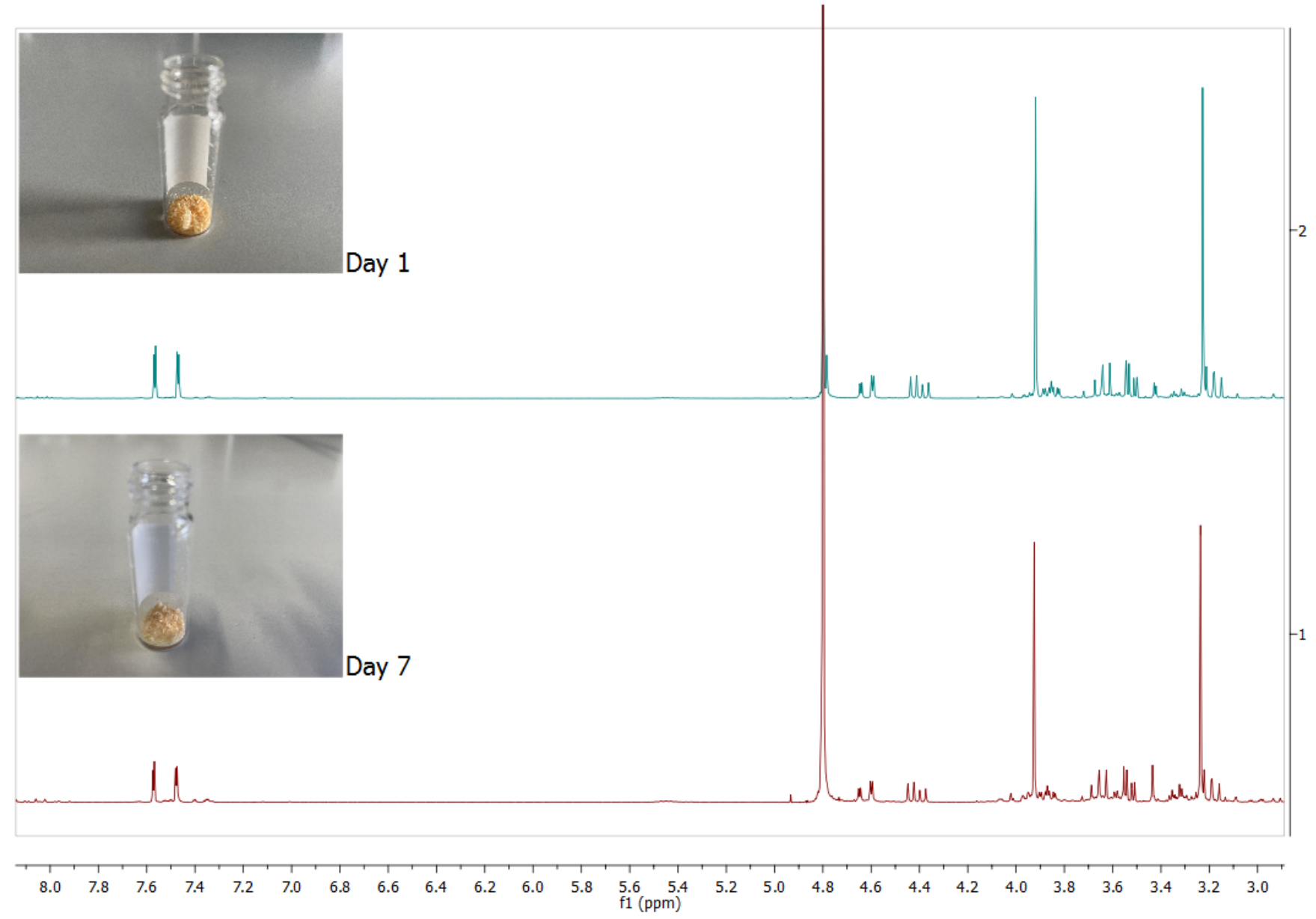

Figure 4. Observations on the stability of 4 .

Since the term hygroscopy describes the ad- or absorption of water molecules from air and, in the case of many salts, can be explained by the energetic profit of forming a hydration shell around the ions, we suspect that 4 has strong intramolecular interactions, thus slowing down the hygroscopic effects. For example, the hydroxide anion may be stabilized by forming hydrogen bonds with the several free $\mathrm{OH}$ groups of 4 while also maintaining an ion pair interaction with the imidazolium cation.

To furthermore prove the viability of $\mathbf{4}$ in the synthesis of new CHILs, we performed neutralization reactions of 4 with four structurally diverse natural acids: D-Glucuronic acid $\mathbf{5 a}$, L-alanine $\mathbf{5 b}$, trans-cinnamic acid $\mathbf{5 c}$ and caprylic acid $\mathbf{5 d}$ (Scheme 2). These reactions generally performed smoothly with 91 to $99 \%$ yield. All products were purified with an individual washing procedure, e.g., the excess of caprylic acid was removed by washing with $n$-heptane, while chloroform is suitable for the removal of remaining trans-cinnamic acid.

In these reactions, 4 takes on the role of an organic building block, which are organic functionalized molecules for the synthesis of structures of higher complexity. The reactive functional group is, in this case, the hydroxide anion of $\mathbf{4}$ for the easy assembly of more complex salts such as $\mathbf{6 a}-\mathbf{d}$.

It is also noteworthy that $\mathbf{6 a}$ is, to the best of our knowledge, the first example of a salt where both the cation and the anion are a carbohydrate.

Finally, we measured the melting and decomposition points of all 6 ionic products featured in this work (Table 1). 


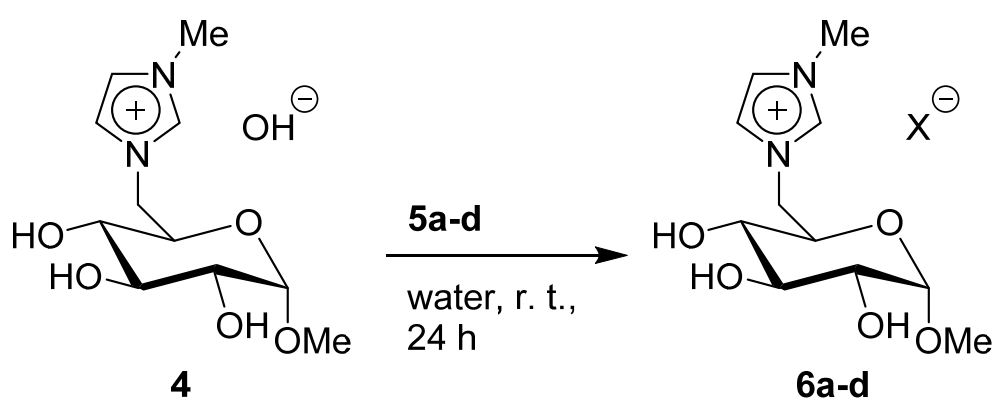<smiles>[X]=C(O)C(O)C(O)C(O)C(=O)[O-]</smiles>

$6 a(98 \%)$<smiles>[X]=C[C@@H](N)C(=O)[O-]</smiles>

6b $(99 \%)$<smiles>[X]#[X]C(=O)/C=C/c1ccccc1</smiles>

$6 c(91 \%)$<smiles>[Y]CCCCCCC(=O)[O-]</smiles>

$6 d(99 \%)$

Scheme 2. Reaction of 4 with several nature-derived acids.

Table 1. Thermal data of our carbohydrate-based salts.

\begin{tabular}{cccc}
\hline Product & Physical State & Melting Point $\left({ }^{\circ} \mathbf{C}\right)$ & Decomposition Point $\left({ }^{\circ} \mathbf{C}\right)$ \\
\hline $\mathbf{3}$ & yellow solid & $172-173$ & 268 \\
$\mathbf{4}$ & yellow solid & $/$ & $139-142^{1}$ \\
$\mathbf{6 a}$ & off-white solid & $106-111$ & 230 \\
$\mathbf{6 b}$ & yellow viscous liquid & $/$ & 238 \\
$\mathbf{6 c}$ & off-white solid & $38-40$ & 275 \\
$\mathbf{6 d}$ & yellow viscous liquid & $/$ & 272 \\
\hline
\end{tabular}

${ }^{1}$ No melting until decomposition.

Out of the given products, $3,4,6 \mathrm{a}$ and $\mathbf{6 c}$ appear as solids at room temperature, while $\mathbf{6 b}$ and $\mathbf{6 d}$ are viscous liquids. The iodide salt 3 has the highest melting point out of the 6 products with $173^{\circ} \mathrm{C}$. This is in line with the common understanding of smaller ions leading to stronger interactions. The hydroxide salt 4 , however, has no measurable melting point until its decomposition. The D-glucuronate salt $\mathbf{6 a}$ is only slightly above the ionic liquid definition with $106-111^{\circ} \mathrm{C}$ and the trans-cinnamate salt $6 \mathrm{c}$ has a very low melting point of $38-40^{\circ} \mathrm{C}$. Thus, the products $\mathbf{6 b}-\mathbf{d}$ can be defined as carbohydrate-based ionic liquids (CHILs).

Looking at their thermal stability, all products but 4 have decomposition points over $230{ }^{\circ} \mathrm{C}$. The D-glucuronate salt $\mathbf{6 a}$ and the L-alaninate salt $\mathbf{6 b}$ are slightly less stable, with decomposition temperatures of 230 and $238^{\circ} \mathrm{C}$, respectively. The iodide (3), trans-cinnamate (6c) and caprylate (6d) salts exhibit slightly higher decompositions points ranging from 268 to $275^{\circ} \mathrm{C}$. All these thermal stability measurements show that even quite distinctively different anions only slightly vary the thermal stability of the whole salt, further proving our previous findings that the carbohydrate cation mostly determines the overall thermal stability [26]. However, it is known from the literature that some anions like OTf, $\mathrm{NTf}_{2}$ or tetracyanoborate may improve the overall thermal stability [27]. The only exception here is 4 , which, most likely due to the reactive nature of the hydroxide anion, decomposes at $139-142{ }^{\circ} \mathrm{C}$.

\section{Conclusions}

In summary, we synthesized a new and surprisingly bench-stable salt containing a glucosylimidazolium cation and a hydroxide anion. This building block can be achieved from commercially available methyl $\alpha$-D-glucopyranoside through a highly optimized 3 -step reaction in $91 \%$ overall yield. We furthermore proved the usability of said building 
block by performing neutralization reactions with four different acids, leading to four new nature-derived salts containing a carbohydrate-based cation in combination with either a carbohydrate-based anion, an amino-acid-based anion, a phenylpropanoid-based anion or a fatty-acid-based anion. Out of the six carbohydrate-based salts synthesized in this work, three are ionic liquids per definition, with two of them doubling as room-temperature ionic liquids.

Supplementary Materials: The following are available online at https:/ /www.mdpi.com/article/10.339 0/compounds1030014/s1: Proton and carbon NMR spectra of all compounds. Figure S1: Methyl-6-iodo- $\alpha-$ D-glucopyranoside 2, Figure S2: 1-(Methyl- $\alpha$-D-glucopyranosid-6-yl)-3-methylimidazolium iodide 3, Figure S3: 1-(Methyl- $\alpha$-D-glucopyranosid-6-yl)-3-methylimidazolium hydroxide 4, Figure S4: 1-(Methyl- $\alpha$-Dglucopyranosid-6-yl)-3-methylimidazolium glucuronate 6a, Figure S5: 1-(Methyl- $\alpha$-D-glucopyranosid-6-yl)3-methylimidazolium alaninate 6b, Figure S6: 1-(Methyl- $\alpha$-D-glucopyranosid-6-yl)-3-methylimidazolium trans-cinnamate 6c, Figure S7. 1-(Methyl- $\alpha$-D-glucopyranosid-6-yl)-3-methylimidazolium caprylate 6d.

Author Contributions: J.S. and S.J. conceived and designed the experiments; J.S. and S.J. analyzed the data; J.S. and S.J. contributed tools; J.S. and S.J. wrote the paper. All authors have read and agreed to the published version of the manuscript.

Funding: This research received no external funding.

Data Availability Statement: All data generated or analyzed during this study are included in this published article.

Conflicts of Interest: The authors declare no conflict of interest.

\section{References}

1. Welton, T. Ionic liquids: A brief history. Biophys. Rev. 2018, 10, 691-706. [CrossRef] [PubMed]

2. Welton, T. Room-Temperature Ionic Liquids. Solvents for Synthesis and Catalysis. Chem. Rev. 1999, 99, 2071-2084. [CrossRef] [PubMed]

3. Eftekhari, A.; Saito, T. Synthesis and properties of polymerized ionic liquids. Eur. Polym. J. 2017, 90, 245-272. [CrossRef]

4. Santos, E.; Albo, J.; Irabien, A. Magnetic ionic liquids: Synthesis, properties and applications. RSC Adv. 2014, 4, 40008-40018. [CrossRef]

5. Jopp, S. Carbohydrate Based Ionic Liquids (CHILs): Synthesis and Applications. Eur. J. Org. Chem. 2020, 2020, 6418-6428. [CrossRef]

6. Oskarsson, A.; Wright, M.C. Ionic Liquids: New Emerging Pollutants, Similarities with Perfluorinated Alkyl Substances (PFASs). Environ. Sci. Technol. 2019, 53, 10539-10541. [CrossRef]

7. Jordan, A.; Gathergood, N. Biodegradation of ionic liquids-A critical review. Chem. Soc. Rev. 2015, 44, 8200-8237. [CrossRef]

8. Flieger, J.; Flieger, M. Ionic Liquids Toxicity-Benefits and Threats. Int. J. Mol. Sci. 2020, 21, 6267. [CrossRef]

9. Reiß, M.; Brietzke, A.; Eickner, T.; Stein, F.; Villinger, A.; Vogel, C.; Kragl, U.; Jopp, S. Synthesis of novel carbohydrate based pyridinium ionic liquids and cytotoxicity of ionic liquids for mammalian cells. RSC Adv. 2020, 10, 14299-14304. [CrossRef]

10. Erfurt, K.; Wandzik, I.; Walczak, K.; Matuszek, K.; Chrobok, A. Hydrogen-bond-rich ionic liquids as effective organocatalysts for Diels-Alder reactions. Green Chem. 2014, 16, 3508-3514. [CrossRef]

11. Jayachandra, R.; Lakshmipathy, R.; Reddy, S.R. Hydrophobic d-galactose based ionic liquid for the sequestration of Pb2+ ions from aqueous solution. J. Mol. Liq. 2016, 219, 1172-1178. [CrossRef]

12. Jayachandra, R.; Reddy, S.R.; Lakshmipathy, R. D-Galactose based hydrophobic ionic liquid: A new adsorbent for the removal of Cd2+ ions from aqueous solution. Environ. Prog. Sustain. Energy 2018, 38, S139-S145. [CrossRef]

13. Courtenay, J.C.; Sharma, R.I.; Scott, J.L. Recent Advances in Modified Cellulose for Tissue Culture Applications. Molecules 2018, 23, 654. [CrossRef]

14. Zhu, H.; Luo, W.; Ciesielski, P.N.; Fang, Z.; Zhu, J.Y.; Henriksson, G.; Himmel, M.E.; Hu, L. Wood-Derived Materials for Green Electronics, Biological Devices, and Energy Applications. Chem. Rev. 2016, 116, 9305-9374. [CrossRef]

15. Jin, H.; Zha, C.; Gu, L. Direct dissolution of cellulose in $\mathrm{NaOH} /$ thiourea/urea aqueous solution. Carbohydr. Res. 2007, 342, 851-858. [CrossRef]

16. Abe, M.; Kuroda, K.; Ohno, H. Maintenance-Free Cellulose Solvents Based on Onium Hydroxides. ACS Sustain. Chem. Eng. 2015, 3, 1771-1776. [CrossRef]

17. Vitz, J.; Erdmenger, T.; Haensch, C.; Schubert, U.S. Extended dissolution studies of cellulose in imidazolium based ionic liquids. Green Chem. 2009, 11, 417-424. [CrossRef]

18. Javed, F.; Ullah, F.; Akil, H.M. Synthesis, characterization and cellulose dissolution capabilities of ammonium-based room temperature ionic liquids (RTILs). Pure Appl. Chem. 2017, 90, 1019-1034. [CrossRef] 
19. Hayouni, S.; Robert, A.; Ferlin, N.; Amri, H.; Bouquillon, S. New biobased tetrabutylphosphonium ionic liquids: Synthesis, characterization and use as a solvent or co-solvent for mild and greener Pd-catalyzed hydrogenation processes. RSC Adv. 2016, 6, 113583-113595. [CrossRef]

20. Jayachandra, R.; Reddy, S.R. Synthesis of D-ribose and D-galactose derived chiral ionic liquids as recyclable chiral solvent for michael addition reaction. Trends Carbohydr. Res. 2015, 7, 60-67.

21. Van Buu, O.N.; Aupoix, A.; Hong, N.D.T.; Vo-Thanh, G. Chiral ionic liquids derived from isosorbide: Synthesis, properties and applications in asymmetric synthesis. New J. Chem. 2009, 33, 2060-2072. [CrossRef]

22. Madsen, R.; Skaanderup, P.R.; Poulsen, C.S.; Hyldtoft, L.; Jørgensen, M.R. Regioselective Conversion of Primary Alcohols into Iodides in Unprotected Methyl Furanosides and Pyranosides. Synthesis 2002, 2002, 1721-1727. [CrossRef]

23. CCDC 2121301 Contains Supplementary Crystallographic Data for This Paper. Available online: https:/ / www.ccdc.cam.ac.uk/ structures /Search?ccdc=2121301 (accessed on 11 October 2021).

24. Seiler, E.R.D.; Takeoka, Y.; Rikukawa, M.; Yoshizawa-Fujita, M. Development of a novel cellulose solvent based on pyrrolidinium hydroxide and reliable solubility analysis. RSC Adv. 2020, 10, 11475-11480. [CrossRef]

25. Yuen, A.K.; Masters, A.F.; Maschmeyer, T. 1,3-Disubstituted imidazolium hydroxides: Dry salts or wet carbenes? Catal. Today 2013, 200, 9-16. [CrossRef]

26. Komabayashi, M.; Nokami, T.; Jopp, S. From Chitin to CHILs: First Glucosamine based Ionic Liquids. Asian J. Org. Chem. 2020, 9 , 2092-2094. [CrossRef]

27. Brzęczek-Szafran, A.; Gaida, B.; Blacha-Grzechnik, A.; Matuszek, K.; Chrobok, A. Bioderived Ionic Liquids and Salts with Various Cyano Anions as Precursors for Doped Carbon Materials. Int. J. Mol. Sci. 2021, 22, 10426. [CrossRef] 